\title{
Unresolved grief in parents of children with CF: A pilot randomised controlled trial on the use and delivery sequence of disease-related education and psychotherapeutic support
}

André Schultz ( $\square$ Andre.Schultz@health.wa.gov.au )

Perth Children's Hospital https://orcid.org/0000-0002-2000-8910

Andrea Barrett

Perth Children's Hospital

Wesley Billingham

Telethon Kids Institute

Cindy Branch-Smith

Telethon Kids Institute

Elizabeth Balding

Telethon Kids Institute

Zubin Grover

Perth Children's Hospital

Gisele Yikilmaz

Telethon Kids Institute

Crystal Bourke

Perth Children's Hospital

Julie Depiazzi

Perth Children's Hospital

Nicole Sander

Perth Children's Hospital

Juliet Foster

Woolcock Institute of Medical Research

Matthew Cooper

Telethon Kids Institute

Florian Zepf

University of Western Australia 
Keywords: Cystic fibrosis, diagnosis, grief, parental response, education, psychotherapy.

Posted Date: July 12th, 2021

DOI: https://doi.org/10.21203/rs.3.rs-669681/v1

License: (c) (1) This work is licensed under a Creative Commons Attribution 4.0 International License. Read Full License 


\section{Unresolved grief in parents of children with CF:A pilot randomised}

\section{2 controlled trial on the use and delivery sequence of disease-related}

\section{3 education and psychotherapeutic support}

5

6 André Schultz ${ }^{1,2,3}$ (andre.schultz@health.wa.gov.au), Andrea Barrett ${ }^{3}$ (andrea.barrett2@ health.wa.gov.au),

7 Wesley Billingham ${ }^{1}$ (wesley.billingham @ telethonkids.org.au), Cindy Branch-Smith ${ }^{1}$ ( $\underline{\text { cindy.branch- }}$

8 smith@telethonkids.org.au), Elizabeth Balding ${ }^{1}$ (elizabeth.balding@ health.wa.gov.au), Zubin Grover ${ }^{3}$

9 (zubin.grover@telethonkids.org.au), Gisele Yikilmaz ${ }^{1}$ (gisele.yikilmaz@health.wa.gov.au), Crystal Bourke ${ }^{3}$ (crystal.bourke@ health.wa.gov.au), Julie Depiazzi ${ }^{3}$ (julie.depiazzi@health.wa.gov.au), Nicole Sander ${ }^{3}$ (nicole.sander@health.wa.gov.au) , Juliet Foster ${ }^{4}$ (juliet.foster@kcl.ac.uk), Matthew Cooper ${ }^{1}$ (matt.cooper@ telethonkids.org.au) , Florian Zepf $\mathbf{f}^{1,2,5,6}$ (florian.zepf@uwa.edu.au)

${ }^{l}$ Wal-yan Respiratory Research Centre, Telethon Kids Institute, University of Western Australia, Perth,

Australia

${ }^{2}$ Division of Paediatrics and Child Health, Faculty of Medicine, University of Western Australia, Perth,

Australia

${ }^{3}$ Perth Children's Hospital, Perth, Western Australia, Australia

${ }^{4}$ Woolcock Institute of Medical Research

${ }^{5}$ Department of Child and Adolescent Psychiatry, Psychosomatic Medicine and Psychotherapy, Jena University

Hospital, Friedrich Schiller University Jena, Germany

${ }^{6}$ Centre and Discipline of Child and Adolescent Psychiatry, Psychosomatics and Psychotherapy, Division of

Psychiatry

\& Clinical Neurosciences, School of Medicine, Faculty of Health and Medical Sciences, University of Western

Australia, Perth, Australia

Corresponding author: André Schultz (andre.schultz@health.wa.gov.au 
Diagnosis of chronic disease in a child can result in unresolved grief (UG) in parents. This study aimed to evaluate the efficacy of psychological insight-oriented therapy (IOT) as a treatment for UG compared to disease related education in parents of children with cystic fibrosis (CF). Sequence of delivery, first IOT then disease related education (or vice versa) was also examined, to let all participants experience both interventions.

Parents were screened for UG. Parents with UG were randomised to either five one-hour sessions of IOT or five one-hour sessions of education. Measures were assessed preintervention, after the first intervention period (primary efficacy assessment), and after the second intervention period (swapping intervention).

Forty-seven parents were screened of which 46.8\% (22/47) had UG. Median duration of UG was 5 years (range: 6 months to 14 years). Anxiety (50\% vs. 20\%, $p=0.03)$ and stress (59\% vs. $28 \%, p=0.03$ ) were significantly more prevalent in parents with UG. There was no difference between arms in the odds of UG resolving either following the first intervention period (OR $0.88 ; 95 \% \mathrm{CI} 0.5,1.5)$ or the second intervention period (OR $0.91 ; 95 \% \mathrm{CI} 0.5$, 1.6). While not statistically significant, adjusted mean values of all nine mental health measures were lower in the IOT (first) arm compared to the ED (first) arm, following the first intervention period.

47 Conclusions: UG is a significant burden for families affected by CF. Provision of disease related education and psychological support, regardless of sequence, can result in resolution 49 of grief. retrospectively registered. 
Cystic fibrosis, diagnosis, grief, parental response, education, psychotherapy.

\section{INTRODUCTION}

56 Cystic Fibrosis (CF) is an autosomal genetic and life limiting disorder that affects approximately 70,000 people $[1 ; 2]$. In many countries, $\mathrm{CF}$ is commonly diagnosed through newborn screening [3]. The news of a CF diagnosis usually is a shock for families and coincides with an important period of laying down the early foundations for parent-child attachment[4], thereby potentially compromising these important relational bonds.

Parents report the period directly following diagnosis to be a time of shock, disbelief, grief [5; 6], heightened emotional experiences and difficult thoughts [7]. This is also typically a time of intense CF related education and engagement with a multidisciplinary team. For some parents the initial disease related education has been found to be an overwhelming experience in which coping capacities can vary greatly $[7 ; 8]$.

67

Parents may dissociate or push away the information about diagnosis during this particular period of crisis and struggle to reach a stage of resolution or acceptance about the diagnosis [9]. The emotional process of resolution requires an acceptance of the emotional pain and other feelings that arise with these news, as well as the acceptance of the long-term aspect of parenting a child with these challenges [9]. As the features of being unresolved are closely associated and parallel with many features of grieving, for the purposes of this study, we will refer to a lack of resolution as unresolved grief (UG) 
76 Prolonged UG can have long-lasting effects on the quality of the parent-child relationship in

77 terms of the parent-child attachment, and consequently on the child's developing sense of self

$78[10 ; 11]$. Specific and targeted interventions to address parental unresolved grief following a

79 diagnosis of CF have not been studied to-date.

80

81 Insight oriented psychotherapy (IOT) aims to help patients develop new insights about their

82 suffering, and to bring about positive change in a person's internal world and state of mind.

83 In the context of potential UG in parents of children with diagnosed CF, IOT on the parental

84 level could be a valuable intervention for affected families.

86 We aimed to:

87 1. Identify (and quantify) a lack of resolution of UG in parents, following their child's diagnosis with $\mathrm{CF}$

2. Evaluate the effectiveness of IOT compared to disease related education in treating UG

3. Examine the sequence of delivery of IOT followed by disease related education (or vice versa) in treating $\mathrm{UG}$

4. Examine the impact of IOT and ED on anxiety, depression and stress.

\section{METHODS}

Study design

96 In this pilot study, participants were screened for UG. Those with UG were then randomised

97 to a pre-post design to assess two interventions. 
99 Parents of children with $\mathrm{CF}$ aged 6 months to 18 years and living within, or close to, the 100 Perth (Australia) metropolitan area were identified through the only paediatric CF service in Western Australia and approached with or mailed information about the study. In total 105 families were approached and 7 opted out of being contacted further by the study team. Parents who gave written informed-consent to participate in the study were screened for grief in relation to their child's diagnosis with $\mathrm{CF}$ with the Reaction to Diagnosis Interview (RDI) [12] administered during a one-hour interview with an RDI-trained psychologist. The interview was video recorded and later analysed by a separate investigator who was trained in the analysis of the RDI interview. Parents with UG continued to randomisation, with one parent enrolled per affected family.

\section{Randomisation}

Following enrolment, participants were randomised to receive either IOT (IOT arm) or education (education arm). Randomisation was carried out by a third party (hospital pharmacy) using randomised blocks of four.

Following the first period of intervention, participants had a one-to-five week break, depending on participant availability, before moving on to the alternate intervention. The decision to give each participant the opportunity to experience both interventions was based on recommendations from clinicians in the hospital's CF service who felt it best all participants were able to experience the IOT component of the research as parents of patients had no other means of direct access to such services through the paediatric hospital setting. Participants were assessed again for UG and emotional wellbeing following each period of intervention. Therefore, participants were assessed for UG and emotional wellbeing at three 
time-points pre (screening), post (after intervention one), and follow-up (after intervention two).

\section{Interventions}

Intervention One (insight-oriented therapy; IOT): Comprised five one-hour sessions of IOT spread out within a minimum of five weeks. The aim of short term IOT was to assist parents to gain a deeper understanding of the impact of their UG surrounding their child's diagnosis on their day-to-day coping, their interpersonal relationships, and their distress surrounding the future of their child, with the aim of bringing about psychological change which would lead to a sense of resolution in relation to their child's CF diagnosis.

Intervention Two (disease related education; hereafter 'education'): Comprised five onehour sessions of CF related education spread out over a period of at least five weeks. Education was provided by experienced members of a $\mathrm{CF}$ care team including a paediatric respiratory physician, CF nurse, physiotherapist, gastroenterologist, and dietician, providing one-on-one information sessions about their areas of expertise in CF. The basic framework for each session is provided in online resource 1. Sessions allowed for questions from parents, and the information provided was tailored to parents' needs. The education sessions aimed to address basic CF related information that parents may not have been receptive to at initial meetings with the clinical CF care team shortly after having been informed about the diagnosis, and that may not have been re-visited with subsequent routine clinical care visits. The overall setting of education sessions could be described as optimistic and caring. 
Resolution of UG formed the primary outcome measure. Other assessed measures of emotional wellbeing included anxiety, depression, and stress, measured using the Depression, Anxiety, Stress Scale (DASS [13]), and the Parent Stress Index (PSI [14]).

Data analysis

Descriptive statistics were calculated for the full sample and per arm. Basic between-group comparisons (at post and follow-up time-points) were made using a Chi-squared test or a Student's t-test, as appropriate.

The primary analysis of whether UG become resolved, was assessed using a logistic regression model, with an odds ratio $(\mathrm{OR})$ and $95 \%$ confidence interval $(95 \% \mathrm{CI})$ reported for the odds of grief resolving in the IOT arm relative to the ED arm. For the other outcomes (emotional wellbeing measures), data from the end of the first period (post) were analysed using a linear regression ANCOVA framework, where the post-time-point measurement was the dependent variable and the pre-time-point measurement was included as an independent variable in the model. All regression models were adjusted for gender of the parent, age of the parent, duration of grief, and, where appropriate the initial measurement of the dependent variable for the period of analysis.

The secondary analysis of the follow-up time-point data were carried out via two different approaches; firstly, via the same framework as for the primary outcome using only data from the post- and follow-up time-points in place of the pre- and post-time-points (respectively) 
and, secondly, using data from the pre- and follow-up time-points using a 'sequence' variable to quantify overall change based on sequence of interventions.

169 Because of the exploratory nature of this pilot study, the analysis does not focus on the statistical significance of the between arm (intervention) differences and instead focuses on the magnitude of effects and variability; $p$-values are presented for some comparisons as a further quantification of the observations against the underlying hypothesis of no difference between arms. All analyses were carried out in using a reproducible research framework implemented within R [15].

Ethics

The study as evaluated and approved by the West Australian Child and Adolescent Health Service Research Ethics Committee RGS2520.

\section{RESULTS}

The CONSORT diagram is presented in Figure 1. Briefly, 47 parents (42 female) were screened for eligibility. Anxiety was present in 34\% (16/47), depression in 28\% (15/47), and stress in $40 \%(21 / 47)$. Of the 47, 22 (48\%) had UG and were randomised. For these 22, the median duration of UG was 5 years (range: 6 months to 14 years). Compared to the parents without UG, parents with UG had higher levels of anxiety (50\% vs. 20\%, $p=0.03)$ and stress (59\% vs. $28 \%, p=0.03)$. No difference was observed for depression. The remaining results relate to the 22 participants that were randomised, of which twelve were randomised to IOT and ten were randomised to education. Cohort descriptive statistics, by arm, are presented in Table 1; the mean age of parents was 37.4 years, and the parents were predominantly female $(19,86.3 \%)$. 


\section{Primary outcome - Unresolved grief}

192 In the IOT arm, UG became resolved in four (of the 12) parents and remained unresolved in four parents; four parents dropped out of the study before completion of this period of the study. In the education arm, UG resolved in seven (of the 10) parents and remained unresolved in two parents; one parent dropped out of the study before completion of this period of the study. In the primary outcome analysis, those in the IOT arm had a nonsignificant, reduced adjusted odds (OR $0.88 ; 95 \%$ CI $0.5,1.5)$, of their grief resolving by the end of the first intervention period.

Of the eight remaining parents in the IOT arm, UG resolved in an additional two parents and returned in one, following the second period where they accessed the education intervention. Of the nine remaining parents in the education arm, UG resolved in an additional one parent and returned in one, following the second period where they accessed the IOT intervention. Those in the IOT arm had a non-significant, reduced adjusted odds (OR 0.91; 95\% CI 0.5, 1.6), of their grief resolving by the end of both intervention periods.

Mean values for all eight mental health outcomes (scales and totals), were all lower postinvention compared to pre, in both arms (Table 2, Figure 2). This decrease was only statistically significant for DASS-Stress in the IOT arm $(-2.12 ; 95 \%$ CI $-3.57,-0.68)$.

211 At the end of the first intervention period, the IOT arm had lower (though the confidence intervals included the value of no difference) mean values for seven of the eight mental 
health outcomes (DASS-Depression being the exception, mean difference estimate 0.0), see

214 Table 3, Figure 3. The difference estimates ranged in magnitude from -0.6 (95\% CI; -3.3, 2.1) for DASS-Stress to -4.2 for both PSI- Parent-Child-Dysfunctional Interaction and PSI-Total.

\section{Secondary analysis (follow-up; post alternate-intervention)}

218 Mean values for most mental health outcomes (scales and totals), were lower at the follow-up 219 time-point compared to the post time-point, in both arms (Table 2, Figure 2); exceptions being DASS-Anxiety and PSI-Parent-Child-Dysfunctional Interaction in the IOT arm, noting that this was following this cohort's education intervention.

222 At the end of the second intervention period, the IOT arm (who had just received education) had higher (though the confidence intervals included the value of no difference) mean values for six of the eight mental health outcomes (PSI-Defensive Response and PSI-Parent-Child-

Dysfunctional Interaction being the exceptions), see Table 3. The analysis of both intervention periods (with follow-up time-point as the dependent variable, and overall sequence (IOT then education) being the variable of interest) yielded no significant differences for the eight mental health outcomes; five of the eight outcomes had a mean between-sequence difference of $<1.0$ unit in magnitude.

\section{DISCUSSION}

Parental unresolved grief (UG) related to their child being diagnosed with CF was highly prevalent amongst parents, and for some this had been present over a decade post diagnosis. Anxiety and stress were more frequently present in parents with UG. IOT and CF education (in either delivery sequence) appeared to mitigate parental grief and resulted in resolution of 
grief. Whilst increased rates of depression and anxiety and higher levels of stress have been well described in patients with $\mathrm{CF}$ and their caregivers, and UG related to other chronic and permanent conditions has been described [16-20], to our knowledge this is the first study to investigate $\mathrm{UG}$ related to a diagnosis of $\mathrm{CF}$ and also the first clinical trial to investigate methods to facilitate resolution of such grief.

In our study population grief remained unresolved for up to 14 years; this extended duration of grief is similar to grief related to loss of a close family member $[21 ; 22]$, which is also associated with adverse mental health outcomes, insecure attachment [23], and has been demonstrated to associate with adverse effects on the child's developing sense of self [10; 11].

The high percentage of resolution of grief seen following the relatively brief interventions applied in our study is encouraging, and may have implications for other conditions. For example, substantial levels of UG has been reported in parents of children with other permanent conditions such as Down syndrome, autism spectrum disorders, cerebral palsy, epilepsy, and phenylketonuria [16-20].

The signal for resolution of grief was supported by strong signals for improvement of comorbidities. All parents with both unresolved grief and depressive symptoms at screening reported a decrease in depressive symptoms once both interventions were completed. Also, ninety one percent of parents with both unresolved grief and anxiety at screening experienced a reduction in anxiety symptoms once both interventions were completed. Increased rates of depression and anxiety and higher levels of stress have been well described in caregivers of 
children with CF [16-20]. Therefore, improvements in both anxiety and stress levels following resolution of grief was encouraging.

The study had a number of limitations. Firstly, the sample size was limited. However, results clearly showed that grief that had been present for years resolved for multiple participants following the relatively brief study period. For some participants the limited intervention may not have been enough to fully explore the depth of their feelings regarding their child's diagnosis and result in resolution of grief, hence the resolution of grief seen with the limited intervention was encouraging. Secondly, study participants were mostly mothers. The implications of our findings to fathers therefore require further study. Thirdly, only one certified RDI coder was used to analyse the data to make the UG diagnosis. Despite this, the reduction in 'coder assessed UG' coincided with a substantial reduction in mental wellbeing indicators, supporting the study's ability to objectively assess change following intervention.

The present study also demonstrated that revisiting CF related education proved to assist parents to resolve UG. Clearly, offering $\mathrm{CF}$ related education to parents shortly after diagnosis (i.e. during a time of high stress, shock and disbelief) may not be conducive towards the information being taken on board and processed, and might result in distortion of information. Providing parents with an opportunity to revisit CF education after a period of adjustment may enable parents to obtain clarity and reprocess information more accurately.

The measurement of UG using the RDI is both time consuming and costly, it requires study by Sher-Censor et al [24] examines a less costly and more time-effective self-reported 
measure of parental resolution, the Reaction to Diagnosis Questionnaire (RDQ), which assesses parental resolution in relation to the child's diagnosis and looks to show promise.

286 In summary, UG is highly prevalent in parents of children with $\mathrm{CF}$, can last over many years 287 and is associated with mental health comorbidities; however, it can potentially be resolved 288 with education and/or psychotherapy. Results suggest the need to investigate how to improve 289 practices around CF diagnosis and incorporate grief identification and appropriately targeted 290 intervention for parents. These results have potential application for other chronic conditions diagnosed in infancy.

293 ACKNOWLEDGEMENTS: The research was funded by an unrestricted grant from Vertex 294 Pharmaceuticals. Salary support was provided to AS by the National Health and Medical 295 Research Council/Medical Research Future Fund (APP1193796). 
1. (2018) Cystic Fibrosis Foundation [U.S.] Patient Registry.

2. Farrell PM (2008) The prevalence of cystic fibrosis in the European Union. J Cyst Fibros 7:450-453

3. Breuer O, Schultz A, Turkovic L, de Klerk N, Keil AD, Brennan S, Harrison J, Robertson C, Robinson PJ, Sly PD, Ranganathan S, Stick SM, Caudri D (2019) Changing Prevalence of Lower Airway Infections in Young Children with Cystic Fibrosis. American journal of respiratory and critical care medicine 200:590-599

4. Ernst MM, Johnson MC, Stark LJ (2011) Developmental and psychosocial issues in cystic fibrosis. Pediatr Clin North Am 58:865-885

5. Jedlicka-Kohler I, Gotz M, Eichler I (1996) Parents' recollection of the initial communication of the diagnosis of cystic fibrosis. Pediatrics 97:204-209

6. Miller AR, Condin CJ, McKellin WH, Shaw N, Klassen AF, Sheps S (2009) Continuity of care for children with complex chronic health conditions: parents' perspectives. BMC Health Serv Res 9:242

7. Havermans T, Tack J, Vertommen A, Proesmans M, de Boeck K (2015) Breaking bad news, the diagnosis of cystic fibrosis in childhood. J Cyst Fibros 14:540-546

8. Jessup M, Douglas T, Priddis L, Branch-Smith C, Shields L, Arest CF (2016) Parental Experience of Information and Education Processes Following Diagnosis of Their Infant With Cystic Fibrosis Via Newborn Screening. J Pediatr Nurs 31:e233-241

9. Pianta RC, Marvin RS, Britner PA, Borowitz KC (1996) Mothers' resolution of their children's diagnosis: Organized patterns of caregiving representations. Infant Mental Health Journal 17:239-256

10. Stern DBB, NY. (1995) The Motherhood Constellation. A unified view of parent-infant psychotherapy. Taylor and Francis, NY

11. Fonagy P, Gergely, G., Jurist, E, \& Target, M (2004) Affect Regulation, Mentalization and the Development of Self. . Taylor and Francis, NY

12. Pianta RC, Marvin, R.S. (1993) Manual for the Classification of the Reaction to Diagnosis Interview. . University of Virginia, Charlottesville, VA, USA

13. Parkitny L, McAuley J (2010) The Depression Anxiety Stress Scale (DASS). J Physiother 56:204

14. Abidin RR (1997) Parenting Stress Index: A measure of the parent-child system. Evaluating stress: A book of resources. Scarecrow Education, Lanham, MD, US, pp 277-291

15. Team RC (2020) R: A language and environment for statistical computing. . R Foundation for Statistical Computing, Vienna, Austria

16. Oppenheim D, Koren-Karie N, Dolev S, Yirmiya N (2009) Maternal insightfulness and resolution of the diagnosis are associated with secure attachment in preschoolers with autism spectrum disorders. Child Dev 80:519-527

17. Barnett D, Clements M, Kaplan-Estrin M, McCaskill JW, Hunt KH, Butler CM, Schram JL, Janisse HC (2006) Maternal resolution of child diagnosis: stability and relations with child attachment across the toddler to preschooler transition. J Fam Psychol 20:100-107

18. Schuengel C, Rentinck IC, Stolk J, Voorman JM, Loots GM, Ketelaar M, Gorter JW, Becher JG (2009) Parents' reactions to the diagnosis of cerebral palsy: associations between resolution, age and severity of disability. Child: care, health and development 35:673-680 
19. Barnett D, Vondra JI (1999) Atypical attachment in infancy and early childhood among children at developmental risk. I. Atypical patterns of early attachment: theory, research, and current directions. Monogr Soc Res Child Dev 64:1-24; discussion 213220

20. Lord B, Ungerer J, Wastell C (2008) Implications of resolving the diagnosis of PKU for parents and children. Journal of pediatric psychology 33:855-866

21. Bylund-Grenklo T, Furst CJ, Nyberg T, Steineck G, Kreicbergs U (2016) Unresolved grief and its consequences. A nationwide follow-up of teenage loss of a parent to cancer 6-9 years earlier. Support Care Cancer 24:3095-3103

22. Sveen J, Eilegard A, Steineck G, Kreicbergs U (2014) They still grieve-a nationwide follow-up of young adults 2-9 years after losing a sibling to cancer. Psychooncology 23:658-664

23. Lannen PK, Wolfe J, Prigerson HG, Onelov E, Kreicbergs UC (2008) Unresolved grief in a national sample of bereaved parents: impaired mental and physical health 4 to 9 years later. J Clin Oncol 26:5870-5876

24. Sher-Censor E, Ram-On TD, Rudstein-Sabbag L, Watemberg M, Oppenheim D (2020) The reaction to diagnosis questionnaire: a preliminary validation of a new self-report measure to assess parents' resolution of their child's diagnosis. Attachment \& Human Development 22:409-424

(1)

(1)

4

5

66


375 Tab. 1 - Cohort descriptive statistics

\begin{tabular}{|l|c|c|c|}
\hline & IOT $^{\text {a }}$ Arm * & Education Arm * & Full Sample \\
\hline $\mathrm{n}$ & 12 & 10 & 22 \\
\hline $\begin{array}{l}\text { Age of parents in } \\
\text { years median } \\
\text { (range) }\end{array}$ & $36.3(6.8)$ & $38.5(7.9)$ & $37.4(7.2)$ \\
\hline $\begin{array}{l}\text { Gender (female: } \\
\text { male) }\end{array}$ & $10: 2$ & $9: 1$ & $19: 3$ \\
\hline Duration of grief & $5.2(4.4)$ & $5.3(4.8)$ & $5.3(4.5)$ \\
\hline
\end{tabular}

376 aOT - insight oriented psychotherapy

$377 *$ mean $(\mathrm{sd})$, or count

378

379

380

381

382

383

384

385

386

387

388

389

390 
Tab. 2 - Descriptive statistics for the mental health outcome measures

\begin{tabular}{|c|c|c|c|c|c|c|c|}
\hline Assessment & Subscale & IOT $^{\text {a }}$ Pre * & ED $^{\mathbf{b}}$ Pre $^{*}$ & IOT $^{\text {a Post }} *$ & ED $^{\mathbf{b}}$ Post * & $\begin{array}{l}\text { IOT Follow-up } \\
(\text { (after ED })^{\text {b }) *}\end{array}$ & $\begin{array}{l}\text { ED }^{\mathbf{b}} \text { Follow-up } \\
\text { (after IOT) * }\end{array}$ \\
\hline n & & 12 & 10 & 8 & 9 & 8 & 7 \\
\hline \multirow[t]{3}{*}{ DASS } & Stress & $8.3(4.3)$ & $10.2(5.6)$ & $6.0(4.5) * *$ & $8.8(5.3)$ & $5.1(4.2)$ & $5.6(3.9)$ \\
\hline & Depression & $4.1(3.0)$ & $5.2(5.5)$ & $3.0(2.7)$ & $4.4(4.4)$ & $1.5(1.2)$ & $2.0(2.6)$ \\
\hline & Anxiety & $3.8(2.9)$ & $4.3(4.7)$ & $1.1(1.2)$ & $3.2(4.3)$ & $2.4(2.6)$ & $2.9(4.1)$ \\
\hline \multirow[t]{5}{*}{ PSI } & Defensive Response & $21.7(5.3)$ & $21.4(4.3)$ & $18.1(3.3)$ & $20.7(6.2)$ & $17.0(4.8)$ & $18.1(4.2)$ \\
\hline & Parental Distress & $34.3(8.2)$ & $33.0(8.6)$ & $29.2(7.6)$ & $34.1(15.1)$ & $27.6(5.2)$ & $28.9(4.5)$ \\
\hline & $\begin{array}{l}\text { Parent-Child-Dysfunctional } \\
\text { Interaction }\end{array}$ & $19.1(6.5)$ & 23.4 (7.9) & $16.4(4.9)$ & $22.6(7.5)$ & $17.2(3.8)$ & $21.1(4.7)$ \\
\hline & Difficult Child Domain & $31.1(12.0)$ & $31.6(8.3)$ & $25.0(6.5)$ & $30.3(8.2)$ & $24.8(7.9)$ & $27.7(5.9)$ \\
\hline & Total & $84.5(22.7)$ & $101.1(44.6)$ & $70.6(14.5)$ & $87.0(27.0)$ & $69.6(13.1)$ & $77.7(10.1)$ \\
\hline
\end{tabular}

* mean (sd), or count

** $p<0.05$ for paired t-test with pre score 
Tab. 3 - Adjusted mean difference between arms, following the first and second study period and overall

\begin{tabular}{|c|c|c|c|c|}
\hline Assessment & Subscale & $\begin{array}{c}\begin{array}{c}\text { Adjusted IOT } \\
\text { effect } \\
(\text { period 1) }\end{array} \\
\end{array}$ & $\begin{array}{l}\text { Adjusted IOT effect } \\
(\text { period 2) } *\end{array}$ & $\begin{array}{c}\text { Adjusted sequence } \\
\text { effect }(\text { IOT then ED } \\
\text { (periods } 1 \text { and } 2) *\end{array}$ \\
\hline $\mathbf{n}$ & & 17 & 15 & 15 \\
\hline \multirow[t]{3}{*}{ DASS } & Stress & $-0.6(-3.3,2.1)$ & $1.2(-2.0,4.5)$ & $0.5(-3.9,5.0)$ \\
\hline & Depression & $0.0(-2.2,2.3)$ & $0.3(-2.8,3.4)$ & $0.2(-3.2,3.5)$ \\
\hline & Anxiety & $-1.2(-4.4,2.1)$ & $0.8(-4.8,6.4)$ & $1.9(-3.4,7.2)$ \\
\hline \multirow[t]{5}{*}{ PSI } & Defensive Response & $-1.3(-6.8,4.3)$ & $-0.7(-5.2,3.9)$ & $0.3(-2.7,3.4)$ \\
\hline & Parental Distress & $-3.8(-17.5,10.0)$ & $0.0(-6.9,6.9)$ & $0.2(-4.0,4.3)$ \\
\hline & $\begin{array}{l}\text { Parent-Child-Dysfunctional } \\
\text { Interaction }\end{array}$ & $-4.2(-11.9,3.5)$ & $-0.3(-8.3,7.6)$ & $-0.2(-3.9,3.6)$ \\
\hline & Difficult Child Domain & $-3.0(-11.5,5.5)$ & $1.4(-9.0,11.8)$ & $1.6(-2.9,6.0)$ \\
\hline & Total & $-4.2(-21.3,13.0)$ & $0.9(-13.9,15.7)$ & $2.9(-3.4,9.3)$ \\
\hline
\end{tabular}

${ }^{\mathrm{a}} \mathrm{IOT}$ - insight oriented psychotherapy; ${ }^{\mathrm{b}} \mathrm{ED}$ - education

* Beta coefficient for the between group difference (IOT arm relative to education arm) from

399 a linear regression model, using an ANCOVA framework, adjusted for parents age, duration

400 of grief, gender, and measure at the start of the period being analysed 


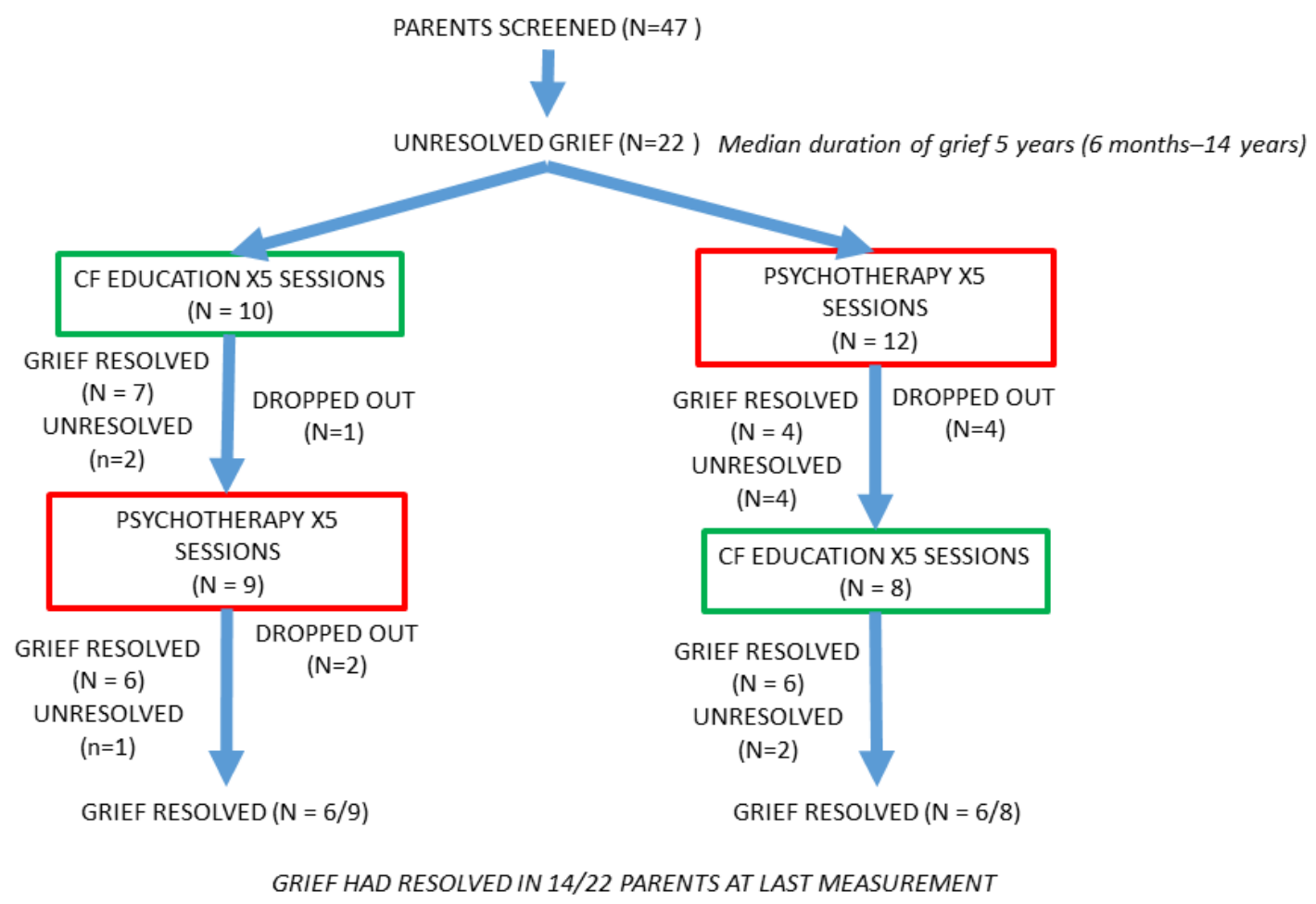


406
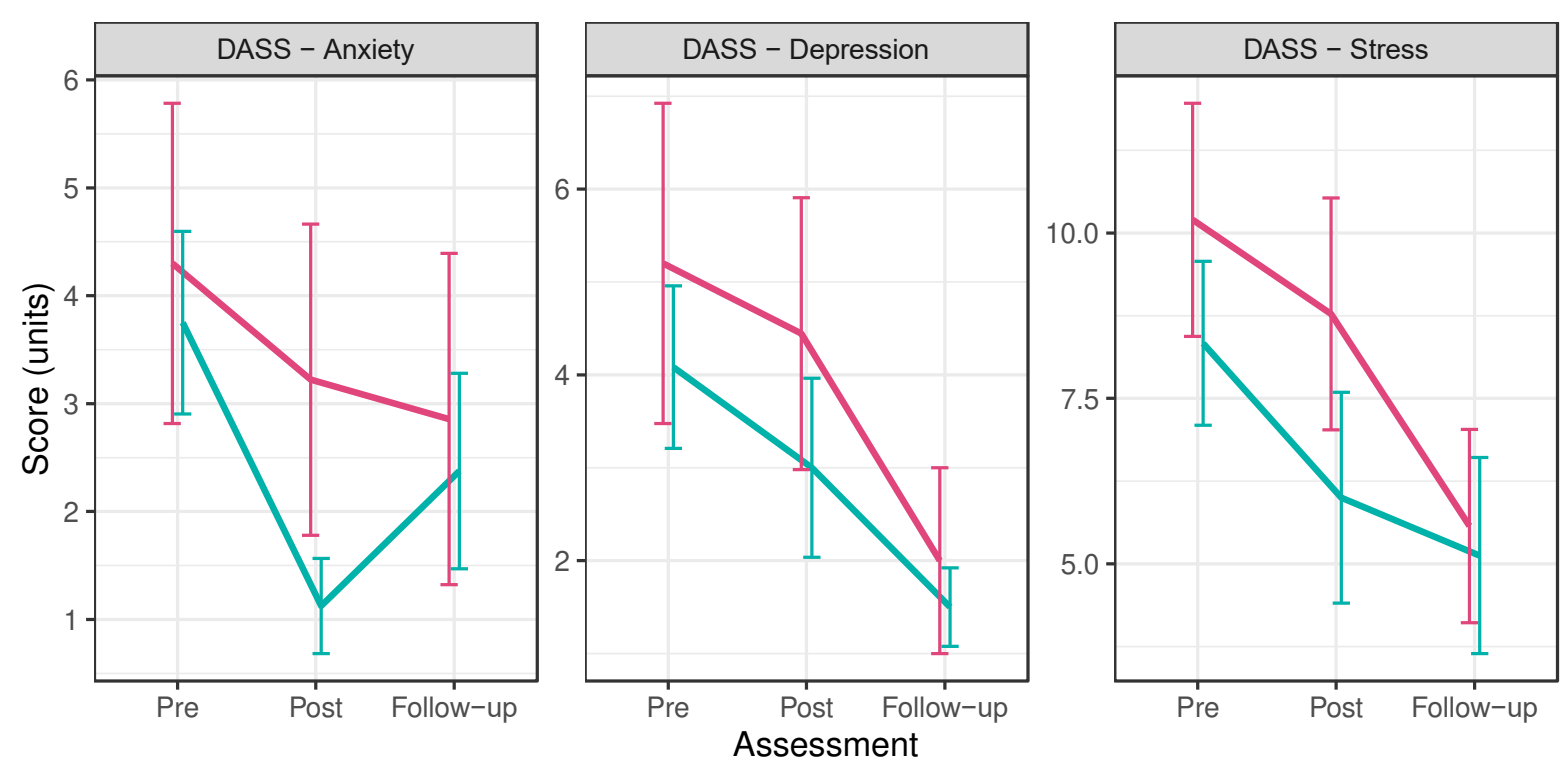

Arm - Education - IOT

407

408

Plotted line is the raw mean of the observed DASS scores (by subscale) from each group;

409

error bars represent one standard error of the mean

410

411

412

413

414

415 
416 Fig. 3 - Adjusted mean difference in measures of emotional wellbeing following 417 intervention

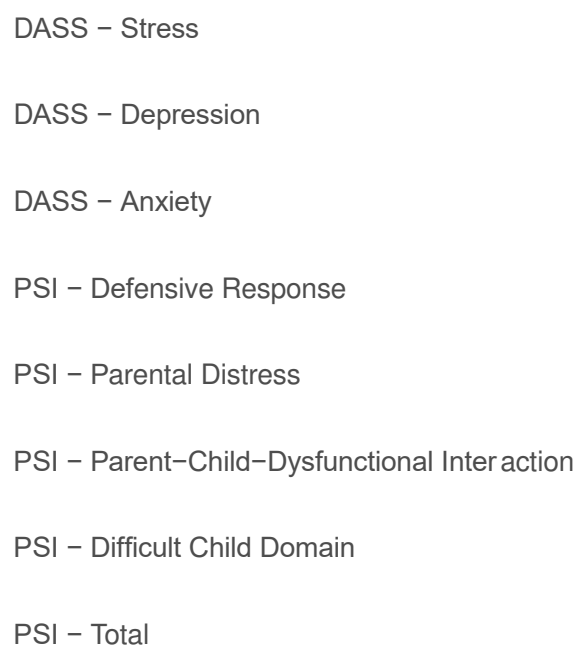

418

419 Plotted point is the adjusted between group difference (IOT arm relative to ED arm) from a 420 linear regression model, using an ANCOVA framework, adjusted for parents age, duration of 421 grief, gender, and measure at the start of the period being analysed; line width represents the $42295 \%$ confidence interval for the adjusted between group difference estimates
$-0.6(-3.3,2.1)$

$0.0(-2.2,2.3)$

$-1.2(-4.4,2.1)$

$-1.3(-6.8,4.3)$

$-3.8(-17.5,10.0)$

$-4.2(-11.9,3.5)$

$-3.0(-11.5,5.5)$

$-4.2(-21.3,13.0)$

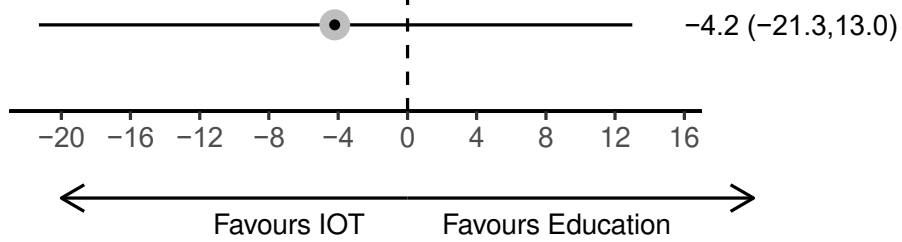




\section{Supplementary Files}

This is a list of supplementary files associated with this preprint. Click to download.

- OnlineResourceEJP.pdf 